\title{
ACCESS TO JOURNALS
}

\author{
HELMUT A. ABT \\ Kitt Peak National Observatory, \\ Box 26732, Tucson, AZ 85726-6732, USA
}

We want to help scientists in developing countries (DC) do research because of their intelligence and devotion to science. But frankly, observational and even theoretical research is very expensive. For a developed country an estimate of the average project cost that includes salaries, the equipment used, and the institutional overhear is roughly $\$ 100,000$ (US). In DC the salaries are lower, but not necessarily the equipment.

How can we help astronomers in DC engage in such expensive activities? I will discuss only the publication and library costs.

I canvassed six journal editors regarding their policies regarding DC and am indebted to them for their honest statements. They were the editors of A\&A, AJ, ApJ, Icarus, MNRAS, and PASP. Three of these have no page charges and the other three generally waive the charges for astronomers in DC, which are defined primarily as India, China, Indonesia, most of South and Central America, eastern Europe, the near east, and Africa except for South Africa.

Regarding subscription rates, journals are distributed to DC either free, at reduced (member) rates, or at full institutional rates. Table 1 gives the distributions.

TABLE 1. Table 1. Subscription Rates to DC for Six Journals

\begin{tabular}{lllllll}
\hline Rates & A\&A & AJ & ApJ & Icarus & MNRAS & PASP \\
\hline free & few & few & few(35) & few & few(40) & few \\
reduced & most & none & most & none & none & none \\
full & none & most & none & most & most & most \\
\hline
\end{tabular}

However it should be said that although AJ and PASP charge full rates, those are less than $\$ 500$ (US) per year.

How large are these rates (including surface postage) for a typical DC? Table 2 shows the data (in US dollars) obtained from one large DC library during three different years for some of its active subscriptions. The numbers marked by colons do not necessarily include postage.

That library has a total budget of $\$ 24,100$ for 1998 , so these nine sets of journals absorbed $59 \%$ of the total.

For financial reasons that library had to terminate subscriptions to the journals listed in Table 3 to which it would have liked to subscribe.

Those six journals would have taken more $(\$ 14,674)$ than the remaining library budget, leaving nothing for books and other astronomy and physics journals.

The reason for the selection of journal subscriptions is easy to guess. If one computes the subscription rate per normalized 1000-word page, the five society publications in Table 2 range from $\$ 0.02$ to $\$ 0.32$ per page while those in Table 3 range from $\$ 2$ to $\$ 25$ per page.

We conclude that the major general astronomical journals have been doing well in aiding research in $\mathrm{DC}$. 
TABLE 2. Table 2. Active Subscriptions in a Typical DC Library

\begin{tabular}{lrrr}
\hline Journal & 1993 & 1996 & 1998 \\
\hline A\&A + A\&AS & $\$ 3108$ & $\$ 4740$ & free \\
AJ & 360 & 405 & $\$ 492$ \\
ApJ + ApJS & 394 & 582 & 664 \\
ApSS & $: 3560$ & 3610 & 3564 \\
Cel. Mechanics & $: 1300$ & 1302 & $: 1302$ \\
Icarus & $: 1760$ & 1975 & 1761 \\
MNRAS & 2100 & free & 3462 \\
PASP + Mercury & 190 & 288 & 349 \\
Solar Physics & 1143 & 2609 & 2628 \\
& & & \\
Total & 13915 & 15511 & 14222 \\
\hline
\end{tabular}

TABLE 3. Table 3. Terminated Subscriptions

\begin{tabular}{lr}
\hline Journal & 1997 Rate \\
\hline Advances in Space Research & $\$ 2024$ \\
Comments on Astrophysics & 1445 \\
Earth, Moon \& Planets & 1205 \\
Fundamentals of Cosmic Physics & 1807 \\
Geophys. \& Astrophys. Fluid Dyn. & 6024 \\
Planetary \& Space Science & 2169 \\
\hline
\end{tabular}

